\title{
Prevalência de periodontite agressiva em adolescentes e adultos jovens do Vale do Paraíba
}

\section{Prevalence of aggressive periodontitis in adolescents and young adults from Vale do Paraíba}

\author{
José Roberto Cortelli* \\ Sheila Cavalca Cortelli** \\ Débora Pallos*** \\ Antonio Olavo Cardoso Jorge****
}

\begin{abstract}
RESUMO: O objetivo do presente estudo foi avaliar a prevalência de periodontite agressiva localizada, periodontite agressiva generalizada e periodontite incipiente em uma população de 15 a 25 anos de idade $(19,4 \pm 3,44)$ da região do Vale do Paraíba - SP que procuraram tratamento odontológico clínico geral no Departamento de Odontologia da Universidade de Taubaté, SP. Seiscentos pacientes, 244 do sexo masculino e 356 do sexo feminino, foram incluídos neste estudo. A condição periodontal da população estudada foi determinada em 6 sítios por dente por meio da avaliação das medidas de profundidade à sondagem e nível clínico de inserção, e confirmada por meio de exame radiográfico. Dez indivíduos $(1,66 \%)$ apresentaram periodontite agressiva localizada, 2 do sexo masculino $(18,5 \pm 2,12)$ e 8 do sexo feminino $(19,2 \pm 3,91), 22(3,66 \%)$ receberam diagnóstico de periodontite agressiva generalizada, sendo 6 do sexo masculino $(19,1 \pm 3,06)$ e 16 do sexo feminino $(20,1 \pm 2,71)$ e $86(14,3 \%)$ foram diagnosticados com periodontite incipiente, 29 do sexo masculino $(20,2 \pm 2,87)$ e 57 do sexo feminino $(21,1 \pm 2,79)$. Houve correlação positiva entre sexo feminino e doença periodontal.

UNITERMOS: Prevalência; Periodontite; Adolescência; Adultos.
\end{abstract}

\begin{abstract}
The aim of the present study was to determine the prevalence of localized and generalized aggressive periodontitis, as well as of incidental attachment loss, in a population of adolescents and young adults aging between 15 and 25 years $(19.4 \pm 3.44)$ from Vale do Paraíba - SP, who searched for general dental care at the Department of Dentistry, University of Taubaté, São Paulo. Six hundred patients, 244 male and 356 female subjects, were included in the studied sample. The periodontal status of this population was evaluated by measuring the depth of periodontal pockets, as well as attachment loss. The data were confirmed by means of radiographic examination. Measurements were performed in six sites per tooth. Ten subjects (1.66\%) were diagnosed as having localized aggressive periodontitis, 2 males (aging $18.5 \pm 2.12$ years) and 8 females (aging $19.2 \pm 3.91$ years); 22 (3.66\%) presented with generalized aggressive periodontitis, 6 males (aging $19.1 \pm 3.06$ years) and 16 females (aging $20.1 \pm 2.71$ years); and 86 individuals (14.3\%) presented with incipient periodontitis, 29 males (aging $20.2 \pm 2.87$ years) and 57 females (aging $21.1 \pm 2.79$ years). There was a positive correlation between the female gender and the occurrence of periodontal disease.
\end{abstract}

UNITERMS: Prevalence; Periodontitis; Adolescence; Adults.

\section{INTRODUÇÃO}

As periodontites agressivas ${ }^{3}$, definidas anteriormente como periodontites juvenis ${ }^{4}$, podem ser clinicamente diagnosticadas e subdivididas em localizadas ou generalizadas. A periodontite agressiva localizada é caracterizada por rápida e severa perda óssea alveolar; os indivíduos afetados podem apresentar pouca quantidade de placa bacteriana e cálculo aderidos sobre as superficies dentárias. A periodontite agressiva generalizada, que mostra pronunciados episódios de destruição periodontal, apresenta sinais mais evidentes de inflamação dos tecidos periodontais e uma maior quantidade de placa bacteriana e cálculo dental em relação à periodontite agressiva localizada ${ }^{19}$. Na periodontite agressiva localizada, os indivíduos apresentam perda de inserção clínica $\geq 4 \mathrm{~mm}$ e os dentes co-

\footnotetext{
*Professor Assistente Doutor de Periodontia da Faculdade de Odontologia da Universidade de Taubaté.

**Aluna do curso de Pós-Graduação (Doutorado) em Bio-Patologia Bucal da Faculdade de Odontologia de São José dos Campos da Universidade Estadual Paulista.

***Aluna do curso de Pós-Graduação (Doutorado) em Pediatria da Universidade Federal de São Paulo.

$* * * *$ Professor Titular de Microbiologia e Imunologia da Faculdade de Odontologia da Universidade de Taubaté e da Faculdade de Odontologia de São José dos Campos da Universidade Estadual Paulista.
} 
Cortelli JR, Cortelli SC, Pallos D, Jorge AOC. Prevalência de periodontite agressiva em adolescentes e adultos jovens do Vale do Paraíba. Pesqui Odontol Bras 2002;16(2):163-168.

mumente atingidos são os primeiros molares e incisivos permanentes, havendo necessariamente $o$ comprometimento de, no mínimo, um primeiro molar. Por outro lado, a periodontite agressiva generalizada caracteriza-se por apresentar perda de inserção clínica $\geq 4 \mathrm{~mm}$ em no mínimo oito dentes permanentes, dos quais, ao menos três dentes, diferem dos primeiros molares e incisivos ${ }^{3}$. Indivíduos que não se enquadram nas peridontites agressivas localizada ou generalizada e apresentam um ou mais dentes com perda de inserção clínica $\geq 3 \mathrm{~mm}$, associada a recessão gengival, injúrias traumáticas, fraturas radiculares, cáries sub gengivais, restaurações iatrogênicas, entre outras, são considerados portadores de periodontite incipiente.

Como áreas isoladas de perda de inserção clínica podem representar um fator de risco para o desenvolvimento de periodontites agressivas, os indivíduos que as apresentam devem ser considerados quando da realização de estudos epidemiológicos ${ }^{1,20}$. Um número expressivo de autores têm realizado estudos sobre prevalência de doença periodontal em indivíduos joven $\mathrm{s}^{1,2,12,13,15,16,17,18} \mathrm{e}$, ao se analisarem esses dados, observa-se variação de 0,1 até $15 \%$ na prevalência de periodontites agressivas localizada e generalizada e periodontite incipiente ${ }^{12,22}$. Essa variação pode ser explicada por diferenças na metodologia empregada, a saber, mensuração dos níveis clínicos de inserção, tamanho da amostra, diferenças ambientais e caracterização da faixa etária estudada. Por outro lado, verifica-se um número reduzido de estudos epidemiológicos ${ }^{7,10,24}$ que caracterizem dados prevalentes das periodontites em adolescentes e adultos jovens no Brasil.

O objetivo deste estudo foi avaliar a prevalência de periodontite agressiva em indivíduos de 15 a 25 anos de idade da região do Vale do Paraíba - SP que procuraram tratamento odontológico clínico geral no Departamento de Odontologia da Universidade de Taubaté.

\section{MATERIAL E MÉTODOS}

Todos os participantes do estudo foram informados quanto ao seu objetivo e, os que concordaram em participar, o fizeram através de autorização. O protocolo deste estudo foi previamente aprovado pelo Comitê de Ética em Pesquisa da Universidade de Taubaté. Foram examinados seiscentos indivíduos de 15 a 25 anos de idade, de am- bos os sexos, por meio de sondagem clínica periodontal. Para a realização deste exame, foram utilizados espelho plano $n^{\circ} 5$, pinça para algodão, sonda exploradora $n^{\circ} 5$ e sonda periodontal milimetrada tipo Willians $n^{\circ} 5$ (HuFriedy). A sondagem clínica periodontal foi realizada em seis pontos, três por vestibular e três pontos por lingual ou palatino em todos os dentes ${ }^{8}$, excluindo-se os terceiros molares, observando-se as medidas de profundidade à sondagem e nível clínico de inserção. As medidas de sondagem clínica periodontal, realizadas por um único examinador*, possibilitaram o estabelecimento do diagnóstico periodontal. Com o objetivo de confirmar o diagnóstico clínico de periodontite, os indivíduos foram submetidos a exame radiográfico. A obtenção de radiografias periapicais, com o emprego de suporte porta-filmes, possibilitou ainda a avaliação do remanescente ósseo alveolar e a presença de dentes inclusos e impactados.

Após a realização da sondagem clínica periodontal e exame radiográfico, foi utilizada fucsina básica a $2 \%$ para evidenciação da placa bacteriana. Todos os participantes do estudo receberam então escova dental macia (Dental Prev) e rolo de fio dental (Dental Prev) para instrução de higiene bucal. De acordo com suas necessidades, cada participante do estudo recebeu tratamento clínico no Departamento de Odontologia da UNITAU.

Os resultados obtidos foram analisados através do teste qui-quadrado, que avaliou de forma isolada a associação entre a prevalência da doença periodontal e o sexo. Ainda, na análise desta associação, foram empregados o odds ratio e o teste exato de Fisher para pequenas amostras. Já para a associação entre a prevalência da doença e idade, foi utilizado o teste $t$ de Student ( $<<0,05)$.

\section{RESULTADOS}

Dos 600 indivíduos examinados, 330 (55\%) mostraram nível clínico de inserção normal (NCI-N) não apresentando assim perda de inserção clínica estabelecida pelos critérios de sondagem periodontal. Estes indivíduos foram caracterizados como portadores de gengivite ou em condições periodontais saudáveis. Cento e cinqüenta e dois indivíduos $(25,5 \%)$ apresentaram ao menos um elemento dentário com perda de inserção clínica de 1 a $2 \mathrm{~mm}$ de profundidade (PIC 1-2 mm). Neste estudo, $10(1,66 \%)$ indivíduos apresentaram características clínicas de periodontite agressiva lo-

*Professor Assistente Doutor de Periodontia - Faculdade de Odontologia da Universidade de Taubaté. 
Cortelli JR, Cortelli SC, Pallos D, Jorge AOC. Prevalência de periodontite agressiva em adolescentes e adultos jovens do Vale do Paraíba. Pesqui Odontol Bras 2002;16(2):163-168.

calizada e $22(3,66 \%)$ de periodontite agressiva generalizada. Oitenta e seis indivíduos (14,3\%) receberam o diagnóstico de periodontite incipiente (Gráfico 1). A Tabela 1 mostra a distribuição da população estudada segundo sexo, média e desvio

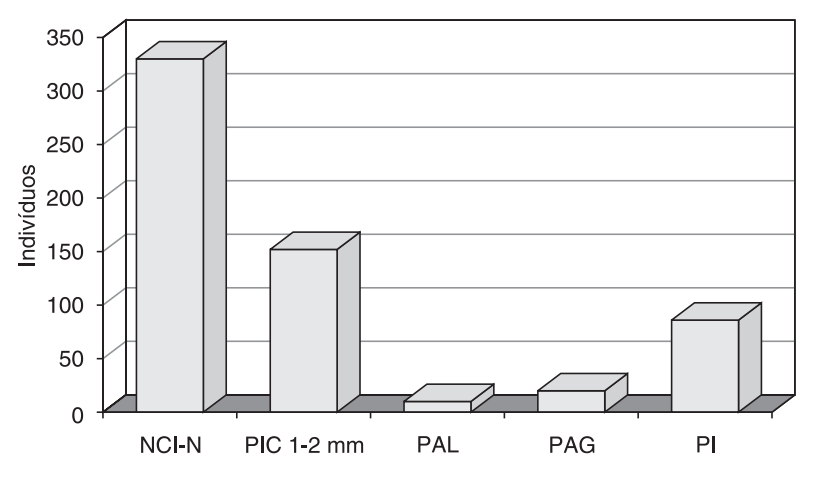

GRÁFICO 1 - Distribuição dos 600 indivíduos examinados de acordo com as condições periodontais. NIC-N = nivel clínico de inserção normal; PIC = perda de inserção clínica; $\mathrm{PAL}=$ periodontite agressiva localizada; $\mathrm{PAG}=$ periodontite agressiva generalizada; $\mathrm{PI}=$ periodontite incipiente. padrão da idade. Os indivíduos foram distribuídos de acordo com o grau de comprometimento dos tecidos periodontais.

Em relação a distribuição dos 118 indivíduos diagnosticados com periodontite agressiva localizada, periodontite agressiva generalizada e periodontite incipiente de acordo com sexo (Tabela 2), foi encontrada associação entre sexo feminino e as diferentes formas de doença periodontal $\left(\chi^{2}=5,27\right.$, $p=0,02, O R=1,65)$. Os dados percentuais encontrados na Tabela 2 estão relacionados com a população total examinada, que corresponde a seiscentos indivíduos.

Assim, o sexo feminino apresentou chance maior em 1,65 vezes, em relação ao sexo masculino, de desenvolver periodontite agressiva localiza$\mathrm{da}$, periodontite agressiva generalizada e periodontite incipiente (Tabela 3).

Quando se compararam, por meio do teste $t$ de Student, as médias de idade entre os indivíduos com periodontite agressiva localizada, periodontite agressiva generalizada e periodontite incipiente, não foram encontradas diferenças estatísticas ao nivel de significância (Tabela 4). O menor valor de $t$ encontra-

TABELA 1 - Distribuição dos 600 indivíduos estudados de acordo com as condições dos tecidos periodontais, divididos por sexo e média de idade.

\begin{tabular}{l|c|c|c}
\hline \hline \multicolumn{1}{c|}{ Grupo } & $\begin{array}{c}\text { Sexo masculino } \\
\mathrm{n}(\text { idade } \pm \mathrm{dp})\end{array}$ & $\begin{array}{c}\text { Sexo feminino } \\
\mathrm{n}(\text { idade } \pm \mathrm{dp})\end{array}$ & $\begin{array}{c}\text { Total } \\
\mathrm{n} \text { (idade } \pm \mathrm{dp})\end{array}$ \\
\hline NCI-N & $134(19,3 \pm 2,80)$ & $196(19,1 \pm 2,44)$ & $330(19,2 \pm 2,64)$ \\
\hline PIC $1-2 \mathrm{~mm}$ & $73(19,7 \pm 2,96)$ & $79(19,8 \pm 3,12)$ & $152(19,7 \pm 3,10)$ \\
\hline PA localizada & $2(18,5 \pm 2,12)$ & $8(19,2 \pm 3,91)$ & $10(19,1 \pm 3,54)$ \\
\hline PA generalizada & $6(19,1 \pm 3,06)$ & $16(20,1 \pm 2,71)$ & $22(19,9 \pm 2,77)$ \\
\hline P incipiente & $29(20,2 \pm 2,87)$ & $57(21,1 \pm 2,79)$ & $86(20,8 \pm 2,86)$ \\
\hline Total & $244(19,1 \pm 3,17)$ & $356(19,6 \pm 3,59)$ & $600(19,4 \pm 3,44)$ \\
\hline \hline
\end{tabular}

NCI-N = nível clínico de inserção normal; PIC = perda de inserção clínica; PA = periodontite agressiva; $\mathrm{P}=$ periodontite; $\mathrm{dp}=$ desvio padrão.

TABELA 2 - Associação entre sexo e prevalência de doença periodontal nos 118 indivíduos diagnosticados com periodontite agressiva localizada, periodontite agressiva generalizada e periodontite incipiente.

\begin{tabular}{l|c|c|c|c|c|c}
\hline \hline \multirow{2}{*}{$\begin{array}{c}\text { Diagnóstico } \\
\text { periodontal }\end{array}$} & \multicolumn{2}{|c|}{ Sexo masculino } & \multicolumn{2}{c|}{ Sexo feminino } & \multicolumn{2}{c}{ Total } \\
\cline { 2 - 7 } & $\mathrm{n}$ & $\%$ & $\mathrm{n}$ & $\%$ & $\mathrm{n}$ & $\%$ \\
\hline PA localizada & 2 & 0,82 & 8 & 2,25 & 10 & 1,66 \\
\hline PA generalizada & 6 & 2,46 & 16 & 4,50 & 22 & 3,66 \\
\hline P incipiente & 29 & 11,88 & 57 & 16,01 & 86 & 14,3 \\
\hline População total & 244 & 40,6 & 356 & 59,3 & 600 & 100 \\
\hline \hline
\end{tabular}

$\mathrm{PA}=$ periodontite agressiva; $\mathrm{P}=$ periodontite. 
Cortelli JR, Cortelli SC, Pallos D, Jorge AOC. Prevalência de periodontite agressiva em adolescentes e adultos jovens do Vale do Paraíba. Pesqui Odontol Bras 2002;16(2):163-168.

TABELA 3 - Associação entre sexo, nível clínico de inserção normal, perda de inserção clínica e tipo de doença periodontal (periodontite agressiva localizada, periodontite agressiva generalizada e periodontite incipiente).

\begin{tabular}{l|c|c|c}
\hline \hline $\begin{array}{c}\text { Diagnóstico } \\
\text { periodontal }\end{array}$ & $\begin{array}{c}\text { Sexo mas- } \\
\text { culino (n) }\end{array}$ & $\begin{array}{c}\text { Sexo femi- } \\
\text { nino (n) }\end{array}$ & $\begin{array}{c}\text { Total } \\
(\mathrm{n})\end{array}$ \\
\hline NCI-N / PIC 1-2 mm & 207 & 275 & 482 \\
\hline PA loc / gen / inc & 37 & 81 & 18 \\
\hline Total & 244 & 356 & 600 \\
\hline \hline
\end{tabular}

$\chi^{2}=5,27, p=0,02, \mathrm{OR}=1,65(1,05-2,59) . \mathrm{NCI}-\mathrm{N}=$ Nivel clínico de inserção normal; PIC = perda de inserção clínica; $\mathrm{PA}$ loc $=$ periodontite agressiva localizada; gen = generalizada; inc $=$ incipiente.

TABELA 4 - Associação entre a média de idade e prevalência de doença periodontal nos 118 indivíduos com periodontite (periodontite agressiva localizada, periodontite agressiva generalizada e periodontite incipiente).

\begin{tabular}{l|c|c|c}
\hline \hline $\begin{array}{c}\text { Diagnóstico } \\
\text { periodontal }\end{array}$ & $\begin{array}{c}\text { Número de } \\
\text { indivíduos }\end{array}$ & $\begin{array}{c}\text { Média de } \\
\text { idade } \pm \mathrm{dp}\end{array}$ & Teste $t$ \\
\hline PA localizada & 10 & $19,1 \pm 3,54$ & $\mathrm{p}>0,05$ \\
\hline PA generalizada & 22 & $19,9 \pm 2,77$ & $\mathrm{p}>0,05$ \\
\hline P incipiente & 86 & $20,8 \pm 2,86$ & $\mathrm{p}>0,05$ \\
\hline \hline
\end{tabular}

$\mathrm{PA}=$ periodontite agressiva $; \mathrm{P}=$ periodontite $; \mathrm{dp}=$ desvio padrão.

do foi 0,64 ao se compararem os grupos periodontite agressiva localizada e periodontite agressiva generalizada e o maior valor, $t=1,37$, foi encontrado ao se compararem os grupos periodontite agressiva generalizada e periodontite incipiente.

\section{DISCUSSÃO}

Uma grande variedade de critérios têm sido utilizados por diferentes autores para determinar índices prevalentes de doença periodontal. Esses critérios incluem diferentes padrões na mensuração de profundidade à sondagem e nível clínico de inserção, obtenção de radiografias periapicais e interproximais como também a seleção das amostras estudadas. Essa diversidade tem mostrado resultados extremamente diferentes na prevalência das doenças periodontais ${ }^{12,14}$.

Dados prevalentes uniformes podem ser conseguidos quando de uma padronização na metodologia utilizada. A obtenção de valores médios de profundidade à sondagem e/ou perda de inserção clínica, associados ou não aos exames radiográficos, pode minimizar essas diferenças ${ }^{12,25}$.

No presente estudo, foi realizada a sondagem clínica periodontal, por um único examinador, para avaliar as medidas de profundidade de sondagem e nível clínico de inserção. Foram utilizados ainda exames radiográficos periapicais, com o propósito de auxiliar o exame clínico, caracterizando-se então o diagnóstico periodontal dos indivíduos examinados. O critério de classificação estabelecido para avaliar a prevalência de periodontite agressiva localizada, periodontite agressiva generalizada e periodontite incipiente foi o definido pela Academia Americana de Periodontia ${ }^{3}$ (1999).

Quando se determina que o critério de profundidade de sondagem e nível clínico de inserção é o ideal para a realização de estudos epidemiológicos, surgem outras questões, como: 1) quantos milimetros de profundidade devem ser considerados para caracterizar as lesões periodontais?; 2) quantos dentes devem ser afetados para se estabelecer um correto diagnóstico periodontal? Baer ${ }^{5}$ (1971) considerou a presença de no mínimo $3 \mathrm{~mm}$ de perda de inserção clínica limitada a dois ou mais primeiros molares ou dois ou mais incisivos, como característica determinante na classificação dos indivíduos com periodontite agressiva localizada. No entanto, Genco et al. ${ }^{9}$ (1986) consideraram o diagnóstico de periodontite agressiva localizada quando da presença de no mínimo $3 \mathrm{~mm}$ de perda de inserção clínica afetando os primeiros molares e incisivos e, adicionalmente, mais um ou dois outros dentes. Estes mesmos autores classificaram os indivíduos com no mínimo $3 \mathrm{~mm}$ de perda de inserção clínica envolvendo mais de 14 dentes como portadores de periodontite agressiva generalizada. Neely ${ }^{17}$ (1992) considerou casos prováveis de periodontite juvenil quando da ocorrência de profundidade à sondagem $\geq 3 \mathrm{~mm}$ atingindo ao menos um primeiro molar. Albandar et al. ${ }^{1}$ (1997) determinaram o diagnóstico de periodontite incipiente nos indivíduos que apresentaram no mínimo a presença de perda de inserção clínica $\geq 3 \mathrm{~mm}$ em um elemento dentário.

A prevalência de periodontite agressiva localizada verificada no presente estudo foi de 1,66\%. Este dado ficou muito acima dos resultados encontrados por outros autores como Löe; Brown ${ }^{12}$ (1991) que observaram 0,53\%, Nassar et al. ${ }^{16}$ (1994) que encontrou $0,42 \%$ e Tinoco et al. ${ }^{24}$ (1997) que relataram prevalência de $0,3 \%$. Todavia, em relação aos achados descritos por Gjermo et al. ${ }^{10}$ (1984), em que, dos 304 indivíduos examinados, oito apresentavam características de periodontite agressiva localizada $(2,63 \%)$, o dado observado neste estudo 
Cortelli JR, Cortelli SC, Pallos D, Jorge AOC. Prevalência de periodontite agressiva em adolescentes e adultos jovens do Vale do Paraíba. Pesqui Odontol Bras 2002;16(2):163-168.

foi inferior. As possiveis razões que podem explicar as diferenças do dado prevalente aqui relatado são possivelmente decorrentes de características regionais e socioeconômicas dos diferentes locais onde os estudos foram realizados. Outras razões que podem ser citadas incluem diferentes critérios de classificação da doença e principalmente a média de idade das populações estudadas, que em geral ficou próxima aos 15 anos de idade. No presente levantamento epidemiológico, foi encontrada uma média de idade próxima aos 20 anos.

A periodontite agressiva generalizada, descrita inicialmente por Page ${ }^{19}$ (1983), caracterizava-se por apresentar lesões periodontais generalizadas sem exibir, no entanto, um padrão de distribuição definido. Além disso, de início entre a puberdade e adolescência apresentava uma rápida e severa destruição óssea alveolar atingindo vários dentes e poderia ainda ser decorrente da periodontite agressiva localizada. No presente estudo, 3,66\% dos indivíduos examinados foram diagnosticados como portadores de periodontite agressiva generalizada. De forma diferente da periodontite agressiva localizada, poucos estudos têm caracterizado especificamente dados prevalentes da periodontite agressiva generalizada e periodontite incipiente. Estes estudos não diferenciaram as periodontites de acordo com o critério proposto pela Academia Americana de Periodontia ${ }^{4,3}$ (1989 e 1999). Assim, os autores utilizaram de forma genérica o termo periodontite de início precoce $e^{1,7,18,23}$ ou periodontite juvenil $^{2,6,15,17,21,25}$ como sinônimos de uma patologia periodontal que atinge adolescentes e adultos jovens com ocorrência de rápida destruição tecidual.

Quando se associa sexo e prevalência de doença periodontal em indivíduos jovens, observa-se uma predileção pelo sexo feminino. Este fato pode ser explicado por uma maior busca de terapia periodontal por mulheres acarretando número elevado de probandos do sexo feminino ${ }^{11}$. Todavia, este fato não é comprovado quando se analisam os descendentes diretos dos probandos, em que se verifica distribuição equivalente da doença periodontal entre indiví-

\section{REFERÊNCIAS}

1. Albandar JM, Brown LJ, Genco RJ, Löe H. Clinical classification of periodontitis in adolescents and young adults. $\mathrm{J}$ Periodontol 1997;68:545-55.

2. Albandar JM. Juvenile periodontitis - pattern of progression and relationship to clinical periodontal parameters. Community Dent Oral Epidemiol 1993;21:185-9.

3. American Academy of Periodontology. International workshop for a classification of periodontal diseases and conditions. Ann Periodontol 1999;4:53-4. duos do sexo feminino e masculino. Neste estudo, foi demonstrada associação entre sexo feminino nos 118 indivíduos com doença periodontal $\left(\chi^{2}=5,27\right.$ e $\left.p=0,02\right)$, o que apontou o sexo como fator de risco para a instalação e desenvolvimento de doença $(O R=1,65)$. Deve-se considerar entretanto que, no presente estudo, foram avaliados apenas os probandos, podendo a análise dos seus descendentes em estudos futuros revelar resultados distintos.

Os indivíduos incluídos no presente estudo apresentavam acesso restrito a cuidados odontológicos e condição socioeconômica limitada, a qual foi previamente estabelecida pelo Serviço de Assistência Social do Departamento de Odontologia da Universidade de Taubaté (UNITAU). A associação destes fatores e a ausência de hábitos de higiene bucal adequados, observados quando da realização dos procedimentos de evidenciação de placa bacteriana, podem ter contribuído para os dados prevalentes observados.

Em função da extensão territorial e diferenças socioeconômicas do País, outros estudos epidemiológicos devem ser conduzidos em diversas regiões, com o objetivo de caracterizar a prevalência das doenças periodontais em adolescentes e adultos jovens.

\section{CONCLUSÕES}

Após a análise dos dados apresentados no presente estudo podemos concluir que:

- a prevalência de periodontite agressiva localizada e generalizada foram respectivamente de $1,66 \%$ e $3,66 \%$, sendo que a prevalência de periodontite incipiente observada foi de $14,3 \%$;

- na população estudada, existiu correlação positiva entre sexo feminino e as patologias periodontais examinadas.

\section{AGRADECIMENTOS}

À Universidade de Taubaté pela concessão de auxílio à pesquisa; a Doctor Smile Cirúrgica e a Dental Prev pelo suprimento de materiais e instrumentais para a realização do estudo.

4. American Academy of Periodontology. Proceedings of the world workshop in clinical periodontics. 1989;1:1-4.

5. Baer PN. The case for periodontosis as a clinical entity. J Periodontol 1971;42:516-20.

6. Barnett ML, Baker RL, Yancey JM. The prevalence of juvenile periodontitis (periodontosis) in a dental school patient population. J Dent Res 1982;61:391-1.

7. Costa FO, Marcos B, Costa JE, Lima LC. Prevalência de doença periodontal de início precoce em crianças e adoles- 
Cortelli JR, Cortelli SC, Pallos D, Jorge AOC. Prevalência de periodontite agressiva em adolescentes e adultos jovens do Vale do Paraíba. Pesqui Odontol Bras 2002;16(2):163-168.

centes de uma escola pública em Belo Horizonte. Rev CROMG 2000;6:53-62.

8. Fetner AE. The complete periodontal examination, diagnosis, and treatment plan. In: American Academy of Periodontology. Periodontal Disease Management. 1994;1: 51-74.

9. Genco RJ, Christersson LA, Zambon JJ. Juvenile periodontitis. Int Dent J 1986;36:168-76.

10. Gjermo P, Bellini HT, Pereira Santos V, Martins JG, Ferracyoli JR. Prevalence of bone loss in a group of Brazilian teenagers assessed on bite-wing radiographs. J Clin Periodontol 1984;11:104-13.

11. Hart TC, Marazita ML, Schenkein HA, Brooks CN, Gunsolley JG, Diehl SR. No female preponderance in juvenile periodontitis after correction for ascertain bias. J Periodontol 1991;62:745-9.

12. Löe H, Brown LJ. Early onset periodontitis in the United States of America. J Periodontol 1991;62:608-16.

13. Lopez NJ, Rios V, Pareja MA, Fernandez O. Prevalence of juvenile periodontitis in Chile. J Clin Periodontol 1001; 8:529-33.

14. MacGregor IDM. Radiographic survey of periodontal disease in 264 adolescent schoolboys in Lagos, Nigeria. Community Dent Oral Epidemiol 1980;8:56-60.

15. Melvin WL, Sandifer JB, Gray JL. The prevalence and sex ratio of juvenile periodontitis in a young racially mixed population. J Periodontol 1991;62:330-4.

16. Nassar MM, Afifi O, Deprez RD. The prevalence of localized juvenile periodontitis in Saudi subjects. J Periodontol 1994;65:698-701
17. Neely AL. Prevalence of juvenile periodontitis in a circumpubertal population. J Clin Periodontol 1992;19:367-72.

18. Oliver RC, Brown LJ, Löe H. Periodontal diseases in the United States population. J Periodontol 1998;69:269-78.

19.

Page RC. Rapidly progressive periodontitis. A distinct clinical condition. J Periodontol 1983;54:197-207.

20. Ranney RR. Classification and epidemiology of periodontal diseases. Periodontology 2000 1993;2:13-25.

21. Saxby MS. Juvenile periodontitis: an epidemiological study in the west Midlands of the United Kingdom. J Clin Periodontol 1987; $14: 594-8$.

22. Saxen L. Juvenile periodontitis. J Clin Periodontol 1980; 7:1-19.

23. Timmerman MF, van der Weijden GA, Armand S, Abbas F, Winkel EG, van Winkelhoff AJ, van der Velden U. Untreated periodontal disease in Indonesian adolescents. Clinical and microbiological baseline data. J Clin Periodontol 1998;25:215-24.

24. Tinoco EM, Beldi MI, Lourenço CA, Lana M, Campedelli F, Tinoco NM, Gjermo P, Preus HR. Localized juvenile periodontitis and A. actinomycetemcomitans in a Brazilian population. Eur J Oral Sci 1997;105:9-14.

25. Van der Velden U, Abbas F, Van Steenbergen TJ, De Zoete OJ, Hesse M, De Ruyter C, De Laat VH, De Graaff J. Prevalence of periodontal breakdown in adolescents and presence of $A$. actinomycetemcomitans in subjects with attachment loss. J Periodontol 1989;60:604-10.

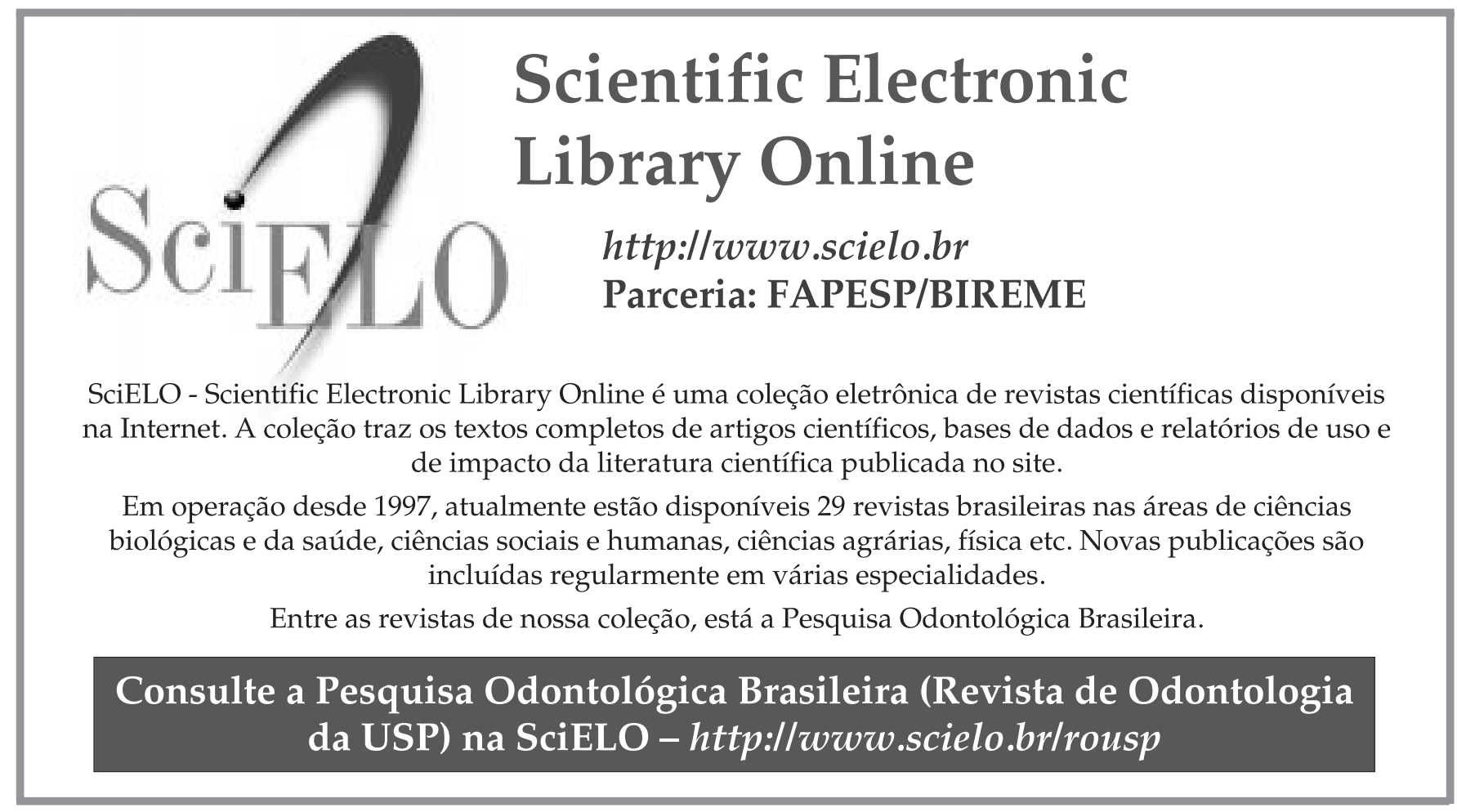

